\title{
„Neue Wege für eine bessere Versorgung“"
}

\author{
Mit innovativen Produkten dem Stigma entgegenwirken
}

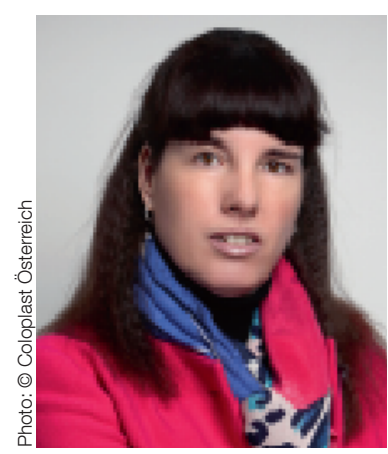

Mag. Michaela Latzelsberger Geschäftsführerin Coloplast Österreich

Die Ursprünge des Unternehmens Coloplast liegen in Dänemark. Dort wurde 1954 der weltweit erste Stomabeutel erfunden und produziert. Die Idee stammte von der dänischen Krankenschwester Elise Sørensen, das Ergebnis war die erste selbsthaftende, dichte Beutelversorgung für Menschen mit einem künstlichen Darmausgang. Coloplast wurde 1957 gegründet und seit 1993 ist der dänische Anbieter auch in Österreich mit einer eigenen Tochtergesellschaft in Wien vertreten. Der Schwerpunkt der Geschäftstätigkeit liegt in den Bereichen Stoma-, Kontinenz- und Wundversorgung. Österreich-Geschäftsführerin Mag. Michaela Latzelsberger erklärt im Gespräch mit procare aktuelle Aktivitäten und Entwicklungen.

\footnotetext{
Der Mensch im Mittelpunkt als Leitmotiv - was heißt das konkret für Coloplast? LATZELSBERGER: Die Mission von Coloplast ist, das Leben von Menschen mit körperlichen Beeinträchtigungen zu erleichtern. Wir sind nahe bei unseren Kunden, wir haben unseren Fokus auf ihre Bedürfnisse gerichtet. Unser Credo: „listen and respond“. Wir hören zu, um ihre Bedürfnisse besser zu verstehen. Wir reagieren, indem wir gemeinsam neue Wege für eine bessere Versorgung finden.

Diskretion, Sicherheit, Lebensqualität wie kommen Coloplast-Produkte diesen Erwartungen entgegen?
}

LATZELSBERGER: Sehr intime, stigmatisierende und in den persönlichsten Bereich gehende körperliche Beeinträchtigungen stehen bei unseren Konsumenten im Vordergrund. Wir versuchen daher Produkte zu entwickeln, die diesem Stigma entgegenwirken, von hoher Qualität und Sicherheit sind und die die Autonomie und Selbstbestimmung des Betroffenen verbessern. An der Entwicklung unserer Produkte sind Betroffene, deren Angehörige, Pflegepersonal und Ärzteschaft beteiligt, denn ihre persönlichen Erfahrungen und Wünsche machen unsere Produkte erst wirklich besonders und innovativ. Daher stehen wir im ständigen Informationsaustausch mit unseren Anwendern. Unsere beiden neuesten Produkte, SpeediCath Flex im Katheter-Bereich und SenSura Mio Original im Stoma-Bereich, wurden zum Beispiel durch den Input von Arbeitsgruppen aus Österreich mitentwickelt.

Inkontinenz und Stoma sind nach wie vor Tabu-Themen - wie geht man damit um: als Firma, als Betroffener und als Betreuer/Pflege?

LATZELSBERGER: Coloplast hat sich als Ziel gesetzt, stets respektvoll in unserem Denken und Handeln umzugehen. Respekt bedeutet für uns, Personen mit Einschränkungen und Behinderung als wertvollen und fähigen Menschen zu begegnen. In unserer Initiative „Stronger Together" wollen wir ein vertrauensvoller Begleiter für alle Beteiligten sein: für Betroffene, deren Angehörige, sowie Fachund Pflegekräfte. Wir hören zu und bieten eine Plattform zum Austausch von Erfahrungen und Ideen, die die Lebensqualität der Betroffenen verbessern können. Wir bringen dabei auch unsere Erfahrung und unser Wissen ein, damit wir zusammen die Bedürfnisse der Betroffenen bestmöglich adressieren können. Indem wir direkt mit Betroffenen und dem Fach- und Pflegepersonal zusammenarbeiten, können wir größere Erfolge erzielen, sowohl sozial, klinisch als auch ökonomisch.

Welchen Stellenwert haben Forschung und Entwicklung bei Coloplast?

LATZELSBERGER: Seit der Gründung vor über 50 Jahren baut Coloplast stetig durch Forschung und innovative Entwicklung eine umfangreiche Produktpalette auf. Wir bieten medizinische Innovationen für die Bereiche Stoma-, Kontinenz-, Wundversorgung und Urologie. Unsere Innovationen sind die Triebfeder von Coloplast und wir versuchen, auch im Design unserer Produkte das „Medizinische“ alltagstauglich erscheinen zu lassen. Wir sind in diesem Bereich auch sehr erfolgreich. Gleich mit vier angesehenen Designpreisen wurde z.B. der SpeediCath ${ }^{\circledR}$ Compact Eve Katheter für Frauen 2015 ausgezeichnet, denn der handliche Katheter vereint Funktionalität mit aufregendem Design und Diskretion.

Wie schaut der Blick in die nächste $\mathrm{Zu}$ kunft von Coloplast aus? Was ist zu erwarten?

LATZELSBERGER: 2017 ist das große Launch-Jahr bei Coloplast Österreich. Mit unserem neuen Männerkatheter SpeediCath Flex setzen wir neue Maßstäbe im Bereich einfache Handhabung und Hygiene beim Selbstkatheterismus. Im Stoma- Bereich setzen wir voll auf SenSura ${ }^{\circledR}$ Mio Original, welches für die Patienten einen flexibleren Tragekomfort und besseren Hautschutz ermöglicht. Es ist mein erklärtes Ziel auch weiterhin jährlich zumindest eine Innovation auf den österreichischen Markt zu bringen.

Was würden Sie sich wünschen - von der Gesellschaft, von der Sozialversicherung, von der Politik - Stichwort: Verschreibungskompetenz der Pflege?

LATZELSBERGER: Ein großer Wunsch ist es, den Zugang zu Innovationen am österreichischen Markt zu erleichtern und vor allem zu beschleunigen. Besonders dann, wenn es gesundheitsökonomisch einen klaren Vorteil für alle Beteiligten bringt. Dies wäre im Interesse der Patienten, die heute leider auf ausgewählte Produktneuheiten länger warten müssen, als einige ihrer europäischen Nachbarn. Auch setze ich viel Hoffnung in das neue Gesundheits-und Krankenpflegegesetz, wodurch es zu einer Anpassung der Berufsbilder in der Pflege an die Herausforderungen im heutigen Versorgungssystem kommen sollte. Eine Entlastung und ein bedarfsorientierterer Einsatz der Pflegekräfte wäre im Sinne der Pflegenden und Ihrer Patienten sehr wünschenswert. 